\title{
Noise and dynamic transitions
}

\author{
G.D. Lythe \\ Department of Applied Mathematics and Theoretical Physics, \\ University of Cambridge, Cambridge CB3 9EW, UK
}

\begin{abstract}
A parabolic stochastic PDE is studied analytically and numerically, when a bifurcation parameter is slowly increased through its critical value. The aim is to understand the effect of noise on delayed bifurcations in systems with spatial degrees of freedom. Realisations of the nonautonomous stochastic PDE remain near the unstable configuration for a long time after the bifurcation parameter passes through its critical value, then jump to a new configuration. The effect of the nonlinearity is to freeze in the spatial structure formed from the noise near the critical value.
\end{abstract}

pp 181-188 in Stochastic PDEs, A. Etheridge (ed) (Cambridge University Press 1994) 


\section{Introduction}

Many physical systems undergo a transition from a spatially uniform state to one of lower symmetry. Such systems are commonly modelled by a simple differential equation which has a bifurcation parameter with a critical value at which there is an exchange of stability between steady states [1]. The model can be improved by adding noise [2], which can be understood as allowing for small rapidly varying effects neglected in the formulation of the model. Noise is also necessary to provide the initial symmetry-breaking which permits the system to choose one of the available lower-symmetry states.

If the bifurcation parameter is not a function of time, the effect of noise is to make a bifurcation ill-defined in an $\mathcal{O}(\epsilon)$ region near the critical value, where $\epsilon$ is the size of the noise [3]. A more dramatic effect is found if the bifurcation parameter is a function of time, corresponding to an experimental situation in which the critical value is not known in advance, so the parameter is slowly changed until a qualitative change is seen in the behaviour of the system [4].

In the case of the pitchfork bifurcation, described by the normal form

$$
\dot{y}=g y-y^{3}
$$

it is normally said that, if $g>0, y$ will be found either at $y=\sqrt{g}$ or $y=-\sqrt{g}$. However, on solving the stochastic differential equation

$$
d y=\left(g y-y^{3}\right) d t+\epsilon d w
$$

with $g=\mu t$, starting with $y=0$ at $g=g_{0}<0$, it is found that $y$ remains near $y=0$ until well after $g=0$ (Figure 1). (The Wiener process - standard Brownian motion - is denoted by lower case $w$.) If $\mu$ is small, a typical trajectory consists of a long sojourn near $y=0$, a sudden jump away from $y=0$ and then relaxation towards $y^{2}=g$. The value of $g$ at which the jump occurs is a random variable whose probability distribution can be found by solving the linearised version of (2) [5]; the

mean value is approximately $\sqrt{2 \mu|\ln \epsilon|}$ and the width of the probability distribution is proportional to $\mu$.

The phenomenon of delayed bifurcation just described and its sensitivity to noise has been studied theoretically and experimentally in the case of non-autonomous stochastic ordinary differential equations [6-9]; the corresponding phenomenon, delayed transition, for PDEs is the subject of this article. A dynamic transition is described by the following non-autonomous parabolic stochastic partial differential equation:

$$
d Y=\left(g(t) Y-Y^{3}+\Delta Y\right) d t+\epsilon d W
$$




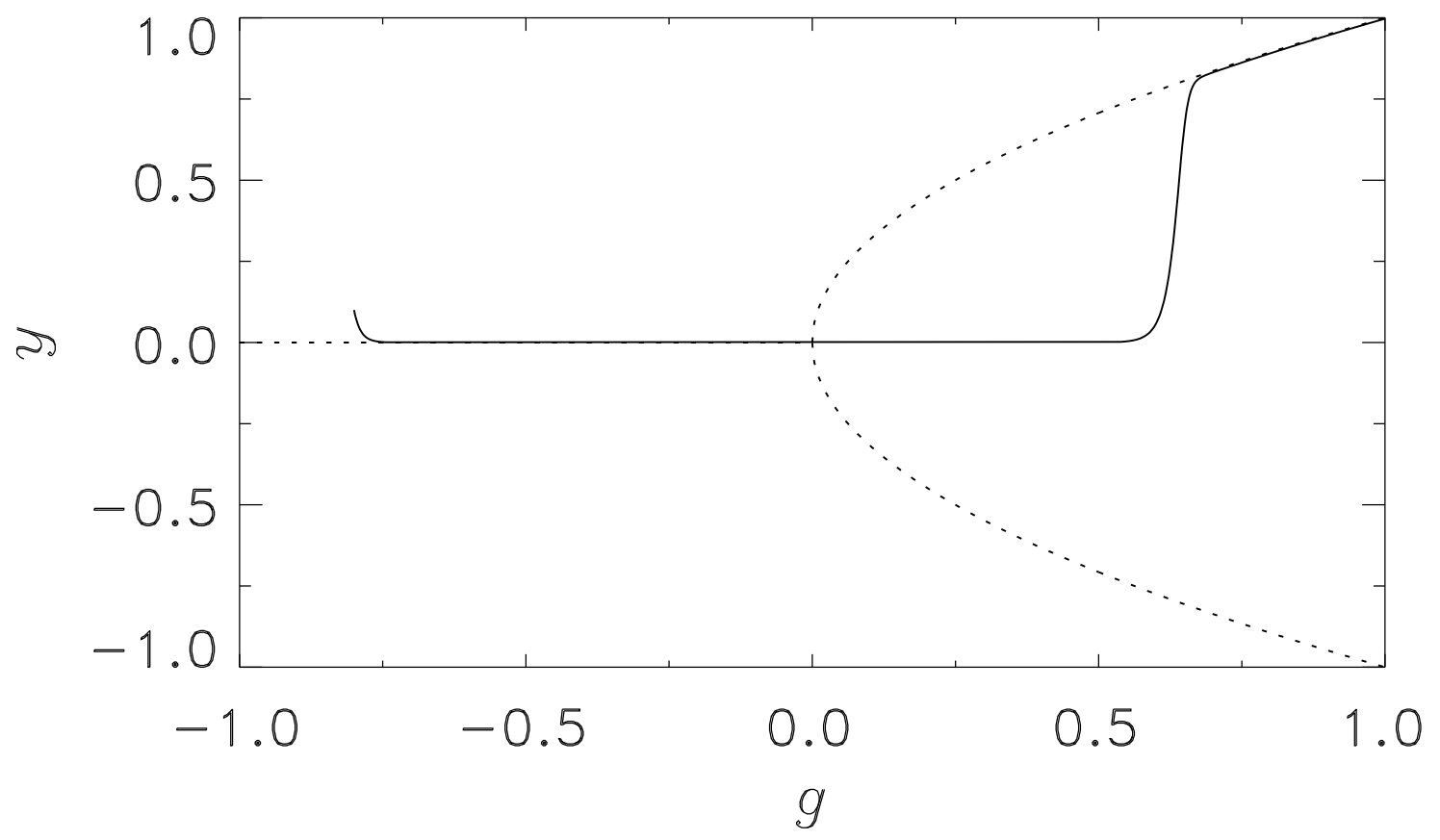

Figure 1. Dynamic pitchfork bifurcation with noise. The solid line is one trajectory of the ordinary $\operatorname{SDE}(2)$, with $\epsilon=10^{-10}, \mu=0.01$, and initial condition $y=0.1$ at $g=-0.8$. Also shown, as dotted lines, are the loci of stable fixed points of the corresponding autonomous system (1). The trajectory lingers near $y=0$ for a long time after $g$ passes through 0 ; the value of $g$ at which the jump towards $y= \pm \sqrt{g}$ occurs is a random variable with mean approximately equal to $\sqrt{2 \mu|\ln \epsilon|}$.

where $g=\mu t$ is slowly increased through $0, W$ is the Brownian sheet, $\Delta=\sum_{i=1}^{m} \frac{\partial^{2}}{\partial x_{i}^{2}}$ is the Laplacian in $\mathbf{R}^{m}$ (the derivatives exist only in the sense of generalised functions) and $\mu$ and $\epsilon$ are constants such that

$$
\epsilon \ll \mu \ll 1 .
$$

In section 2 this initial value problem is solved using periodic boundary conditions in one space dimension, in which case $Y$ is a stochastic process with values in the space of real-valued continuous functions on $[0, L]$, and has continuous mean $\left\langle Y_{t}(x)\right\rangle$ and correlation function $\left\langle Y_{t}(x) Y_{t}\left(x^{\prime}\right)\right\rangle[10,11]$. In section 3 the corresponding equation in space dimension, $m$, greater than one is discussed.

The present work was motivated by the behaviour of some nonlinear systems of ordinary and partial differential equations whose dynamics are controlled by noise $[12,13]$. The controlling influence of noise arises because trajectories spend long times near a slow invariant manifold, and the dynamics near this manifold are similar to those of a dynamic bifurcation [5]. The most important parameter in the 

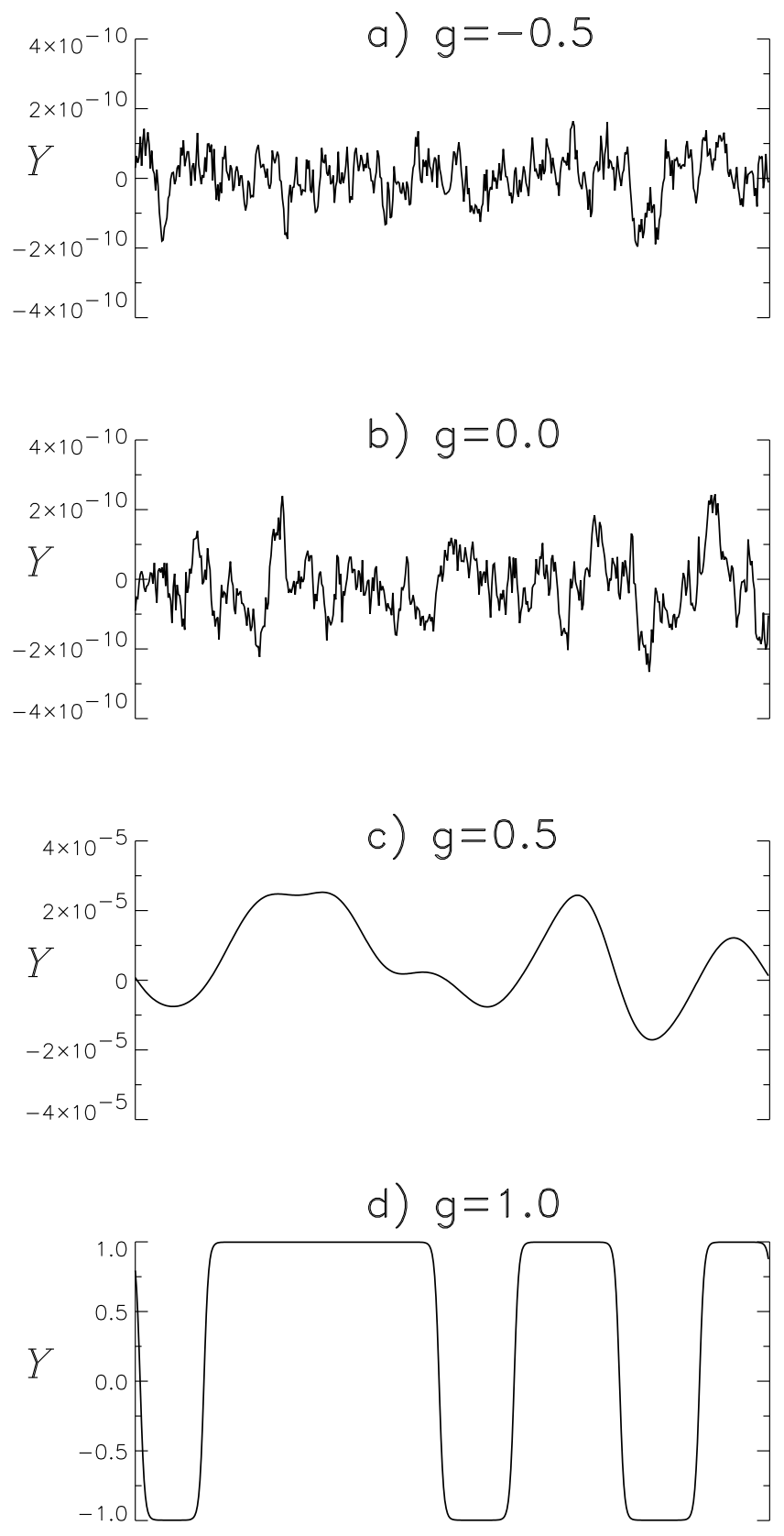

Figure 2. Dynamic transition in one space dimension. One numerically-generated realisation of the stochastic $P D E$ (3) $Y_{t}(x), x \in[0, L]$, is displayed at four times as the bifurcation parameter, $g=\mu t$, is slowly increased, passing through 0 at $t=0$. (Note the different vertical scales.) It remains close to the zero configuration until well after $g=0$. Nonlinear terms become important when $g \simeq \sqrt{2 \mu|\ln \epsilon|}$; their effect is to freeze in the spatial structure formed, near $g=0$, from the noise. The data shown here were generated using a finite difference method with 500 points. $\left(L=300, \mu=0.01, \epsilon=10^{-10}\right.$.)

description of these systems is $\mu|\ln \epsilon|$, where $\frac{1}{\mu}$ is the timescale for the dynamics near the slow manifold and $\epsilon$ is the magnitude of the noise. 


\section{Model dynamic transition in one space dimension}

Four configurations from a numerical realisation of (3) with $m=1$ are shown in Figure 2. According to static stability analysis the zero configuration is unstable for $g>0$, but $Y$ typically remains much closer to 0 than to $Y^{2}=g$ until well after $g=0$ when $g$ is time-dependent. The first part of a dynamic transition, the loss of stability of the zero configuration, is thus controlled by the noise and the sweep rate, not by the nonlinearity.

If $Y$ obeys (3), the linearised version of the stochastic differential equation for its $k$ th Fourier mode, $u_{t}^{(k)}=\frac{1}{\sqrt{L}} \int_{0}^{L} Y_{t}\left(x^{\prime}\right) \mathrm{e}^{\mathrm{i} k x 2 \pi / L} d x^{\prime}$, is

$$
d u^{(k)}=\left(g(t)-\left(\frac{2 \pi}{L}\right)^{2} k^{2}\right) d t+\epsilon d w^{(k)},
$$

with each $w^{(k)}$ an independent Wiener process. Thus, for as long as nonlinear terms are unimportant, each Fourier mode evolves independently, satisfying an equation of the dynamic bifurcation type.

As the in ODE case, the new configuration (Figure 2(d)) appears abruptly at a value of $g$ which is a random variable with mean approximately $\sqrt{2 \mu|\ln \epsilon|}$. The effect of the cubic nonlinearity is to arrest the exponential growth of $\left\langle Y_{t}(x) Y_{t}(x)\right\rangle$ when it becomes $\mathcal{O}(1)$ and to freeze in the spatial structure formed during the slow sweep. Establishing when this happens makes it possible to answer another question which is not considered in the static version: what is the typical size of the spatial domains formed in the transition?

To examine the dynamics of the loss of stability of the zero configuration, consider the solution of the linearised version of (3),

$$
d Y=(g(t) Y+\Delta Y) d t+\epsilon d W,
$$

with initial data $Y_{t}(x)=f(x)$ on $x \in[0, L]$ at $t=t_{0}<0$ when $g=g_{0}$ :

$$
Y(x, t)=\int_{0}^{L} G\left(t, t_{0}, x, x^{\prime}\right) f\left(x^{\prime}\right) d x^{\prime}+\epsilon \int_{t_{0}}^{t} \int_{[0, L]} G\left(t, t_{0}, x, x^{\prime}\right) d W_{t}\left(x^{\prime}\right) .
$$

The first integral is an ordinary integral; the second a space-time Itô integral. The fundamental solution $G\left(t, t_{0}, x, x^{\prime}\right)$ is given by

$$
G\left(t, t^{\prime}, x, x^{\prime}\right)=\frac{1}{\sqrt{4 \pi\left(t-t^{\prime}\right)}} \mathrm{e}^{\frac{1}{2} \mu\left(t^{2}-t^{\prime 2}\right)} \sum_{j=-\infty}^{\infty} \mathrm{e}^{-\frac{\left(x-x^{\prime}-j L\right)^{2}}{4\left(t-t^{\prime}\right)}} .
$$

The solution (7) prescribes the mean and correlation function of $Y$ as a function of time. The mean value of $Y$ is the first term, an ordinary integral which, for large $t-t_{0}$, is given by

$$
\left\langle Y_{t}(x)\right\rangle=\mathrm{e}^{\frac{1}{2} \mu\left(t^{2}-t_{0}^{2}\right)} \bar{y}
$$


where $\bar{y}$ is a smoothed version of the initial data $f(x)$. In the rest of this article, it is assumed that $\bar{y} \leq \mathcal{O}(1)$ and $2 \mu|\ln \epsilon|<g_{0}^{2}$. This ensures that $\left\langle Y_{t}\right\rangle^{2} \ll\left\langle Y_{t}^{2}\right\rangle$, ie $Y$ is taken as a mean-zero Gaussian process. It is also assumed that $\left|t_{0}\right|>\frac{1}{\sqrt{\mu}}$, so that quasi-static equilibrium is reached before $g=0$, and $L \gg \sqrt{t-t_{0}}$ so that, at any one $x$, only one term in (8) is important.

The correlation function is a mean square quantity which can be evaluated using the 'delta function' property of the Brownian sheet as an integrator:

$$
\begin{aligned}
\left\langle Y_{t}(x) Y_{t}\left(x^{\prime}\right)\right\rangle & =\epsilon^{2} \int_{0}^{L} \int_{t_{0}}^{t} G^{2}\left(t, s, x, x^{\prime}\right) d s d x^{\prime} \\
& =\epsilon^{2} \int_{t_{0}}^{t} d s \frac{\mathrm{e}^{\mu\left(t^{2}-s^{2}\right)}}{\sqrt{8 \pi(t-s)}} \mathrm{e}^{-\frac{\left(x-x^{\prime}\right)^{2}}{8(t-s)}} .
\end{aligned}
$$

For times $t$ such that $-t>1 / \sqrt{\mu}$ the nonautonomous correlation function (10) differs only by $\mathcal{O}(\mu)$ from the result obtained for fixed $g<0$ if $t_{0} \rightarrow \infty$ [14]:

$$
\left\langle Y_{t}(x) Y_{t}\left(x^{\prime}\right)\right\rangle=\frac{\epsilon^{2}}{4 \sqrt{|g|}} \mathrm{e}^{-\left|x-x^{\prime}\right| \sqrt{|g|}}
$$

In the non-autonomous case, the correlation function remains finite as $t$ passes through 0 , and for large positive times $(t>1 / \sqrt{\mu})$, it is well approximated by:

$$
\left\langle Y_{t}(x) Y_{t}\left(x^{\prime}\right)\right\rangle \simeq \epsilon^{2} \frac{\mathrm{e}^{\mu t^{2}}}{\sqrt{8 \mu t}} \mathrm{e}^{-\left(x-x^{\prime}\right)^{2} / 8 t}
$$

Note the Gaussian form of the spatial correlation and the existence of a characteristic length $\sqrt{8 t}$ at time $t$. This formula is valid until $g \simeq \sqrt{2 \mu|\ln \epsilon|}$, when the cubic nonlinearity becomes important. The correlation length at this time becomes the characteristic size of the spatial domains formed (Figure 2(d)).

\section{Higher space dimensions}

Proceeding as in section 2, the expression obtained for the correlation function at time $t$ is

$$
\left\langle Y_{t}(x) Y_{t}\left(x^{\prime}\right)\right\rangle=\int_{t_{0}}^{t} d s \frac{\mathrm{e}^{\mu\left(t^{2}-s^{2}\right)}}{(8 \pi(t-s))^{\frac{m}{2}}} \mathrm{e}^{-\frac{\left\|x-x^{\prime}\right\| \|^{2}}{8(t-s)}},
$$

where $\left\|x-x^{\prime}\right\|$ is the Euclidean norm on $\mathbf{R}^{m}$. The major difference from the case $m=1$ is that the correlation function does not approach a finite limit as $\left\|x-x^{\prime}\right\| \rightarrow 0$. This is a general feature of second order stochastic PDEs in more than one space dimension $[15,16]$. On a finite grid, equation (6), with $g<0$ fixed, is of interest because it generates a stochastic process with non-zero correlation length in both space and time [17]. The singularity in the correlation function at 
$x=x^{\prime}$ manifests itself in the normalisation, which increases without a finite limit as the grid spacing is decreased. In the nonautonomous version the same divergence is seen (logarithmic when $m=2$ ), but for $g>0$ the singularity is only noticeable on very small length scales. The nature of these divergences, in both discrete and continuous versions, is conveniently studied in Fourier space, where the linearised equation separates into uncoupled ordinary SDEs, each of which can be solved exactly.

The finite difference method for a parabolic SPDE consists of replacing the infinite dimensional system (3) by $N^{m}$ ordinary SDEs on a grid of equally-spaced points in $[0, L]^{m}$. For the SPDE (3), the SDE at each point is

$$
d Y(x)=\left(g Y(x)-Y^{3}(x)\right) d t+\rho \tilde{\Delta} Y(x) d t+\rho^{\frac{m}{4}} \epsilon d w
$$

where the discrete Laplacian $\tilde{\Delta}$ is defined by

$$
\tilde{\Delta} Y(x)=\sum_{x^{\prime}} Y\left(x^{\prime}\right)-2 m Y(x)
$$

and the sum is over the $2 m$ nearest neighbours of $x$. Each of the $N^{m}$ ordinary SDEs has an associated Wiener process, independent of all the others, and so can be numerically solved in the normal way [18]. Note the scaling of the magnitude of the noise added at each grid point with $\rho$. The smallest length scale resolved is roughly the distance between nearest neighbours, $\frac{1}{\sqrt{\rho}}$. As in numerical solution of deterministic parabolic PDEs, there is a maximum timestep, proportional to $\frac{1}{\rho}$, if the finite difference method is to be numerically stable [19].

Solved on a finite grid in this way, the behaviour of realisations of the nonautonomous version SPDE (3), with $m>1$ and $g=\mu t$ as in section 2, is qualitatively similar to that in one space dimension [20]. That is, a Gaussian form of the spatial correlation function emerges from the slow sweep through $g=0$,

$$
\left\langle Y_{t}(x) Y_{t}\left(x^{\prime}\right)\right\rangle \simeq \epsilon^{2} \sqrt{\frac{\pi}{\mu}} \frac{\mathrm{e}^{\mu t^{2}}}{(8 \pi t)^{\frac{m}{2}}} \mathrm{e}^{-\left\|x-x^{\prime}\right\|^{2} / 8 t}
$$

and metastable domains of positive and negative $Y$ are formed when the nonlinearity becomes important, at $g \simeq \sqrt{2 \mu|\ln \epsilon|}$.

\section{Conclusion}

In systems with spatial degrees of freedom, the phenomenon of delayed bifurcation is accompanied by a noise-controlled formation of spatial structure. Here the stochastic PDE (3) was studied, whose realisations consist long sojourns near the zero configuration during which the shape of the spatial correlation function becomes Gaussian, followed by an abrupt change to the nonlinear régime, with the formation of domains of positive and negative $Y$. This spatial structure, formed near the critical value of the bifurcation parameter $g$ when noise dominates, is frozen in at $g \simeq \sqrt{2 \mu|\ln \epsilon|}$, when the characteristic length is $\sqrt{8 t}$. 


\section{References}

[1] Alan C. Newell, Thierry Passot and Joceline Lega "Order parameter equations for patterns." Ann. Rev. Fluid Mech. 25 399-453 (1993).

[2] Frank Moss and P.V.E. McClintock, Noise and nonlinear dynamical systems (Cambridge University Press, Cambridge, 1987).

[3] C. Meunier and A.D. Verga "Noise and Bifurcations." J. Stat. Phys. 50 345375 (1988).

[4] Christopher W. Meyer, Guenter Ahlers and David S. Cannell "Stochastic influences on pattern formation in Rayleigh-Bénard convection: Ramping experiments." Phys. Rev. A 44 2514-2537 (1991).

[5] G.D. Lythe and M.R.E. Proctor "Noise and slow-fast dynamics in a three-wave resonance problem." Phys. Rev. E. 47 3122-3127 (1993).

[6] N.G. Stocks, R. Mannella and P.V.E. McClintock "Influence of random fluctuations on delayed bifurcations: The case of additive white noise." Phys. Rev. A 40 5361-5369 (1989).

[7] J.W. Swift, P.C. Hohenberg and Guenter Ahlers "Stochastic Landau equation with time-dependent drift." Phys. Rev. A 43 6572-6580 (1991).

[8] Claude Lobry "A propos du sens des textes mathématiques. Un example: la théorie des "bifurcations dynamiques"." Ann. Inst. Fourier 42 327-352 (1992).

[9] V.I. Arnol'd (Ed.), Dynamical Systems V (Springer, Berlin, 1994).

[10] Tadahisa Funaki "Random motion of strings and related stochastic evolution equations." Nagoya Math. J. 89 129-193 (1983).

[11] I. Gyöngy and E. Pardoux "On quasi-linear stochastic partial differential equations." Probab. Theory Relat. Fields 94 413-426 (1993).

[12] M.R.E. Proctor and D.W. Hughes "The false Hopf bifurcation and noise sensitivity in bifurcations with symmetry." Eur.J.Mech.,B/Fluids 10 81-86 (1990).

[13] Miltiades Georgiou and Thomas Erneux "Pulsating laser oscillations depend on extremely-small-amplitude noise." Phys. Rev. A 45 6636-6642 (1992).

[14] H. Haken, Synergetics (Springer, Berlin, 1978).

[15] J.B. Walsh (1986) "An introduction to stochastic partial differential equations," in Ecole d'été de probabilités de St-Flour XIV, P.L. Hennequin (Ed.). Springer: Berlin.

[16] Charles R. Doering "Nonlinear Parabolic Stochastic Differential Equations with Additive Colored Noise on $\mathbf{R}^{d} \times \mathbf{R}_{+}$: A Regulated Stochastic Quantization." Commun. Math. Phys. 109 537-561 (1987).

[17] J. García-Ojalvo and J.M. Sancho "Colored noise in spatially extended systems." Phys. Rev. E 49 2769-2778 (1994).

[18] Peter E. Kloeden and Eckhard Platen, Numerical Solution of Stochastic Differential Equations (Springer, Berlin, 1992).

[19] Leon Lapidus and George F. Pinder, Numerical Solution of Partial Differential Equations in Science and Engineering (Wiley, New York, 1982).

[20] G.D. Lythe, Stochastic slow-fast dynamics (Ph.D. thesis, University of Cambridge, 1994). 